\title{
Navigating Turn-Taking and Conversational Repair in an Online Synchronous Course
}

\author{
Yvonne Earnshaw \\ Beaverton, Oregon
}

\begin{abstract}
In face-to-face conversations, speaker transitions (or hand-offs) are typically seamless. In computer-mediated communication settings, speaker hand-offs can be a bit more challenging. This paper presents the results of a study of audio communication problems that occur in an online synchronous course, and how, and by whom, those problems are resolved. Data were collected from chat transcripts and audio transcripts from a graduate level discourse and conversation analysis course that used WebEx, an audio conferencing software application that also has a chat channel. Using a conversation analysis approach, data were analyzed to identify when speaker hand-offs occurred to determine related patterns of confirmation strategies and repair sequences. Findings showed several different approaches to smooth speaker hand-offs. In cases where handoffs were not smooth, participants attempted corrections by either fixing the problem or by moving on. There were also instances in which parties encountered technical difficulties with the audio or with Internet connectivity. Parties used the chat channel to indicate that they were having trouble. The instructor's role was to troubleshoot, call upon students, and move the discussion along. This study provides some insight on how chat can be used in a discussion-based, online synchronous course to identify technical difficulties when a speaker is called upon and how the difficulty is resolved.
\end{abstract}

Keywords: synchronous, audio conference, repair, conversation analysis, turn-taking, CMC

Earnshaw, Y. (2017). Navigating turn-taking and conversational repair in an online synchronous course. Online Learning, 21(4), 315-336. doi: 10.24059/olj.v21i4.1029

\section{Navigating Turn-Taking and Conversational Repair in an Online Synchronous Course}

There has been an increase in the use of synchronous communication and collaboration tools, such as audio conferencing, video conferencing, and synchronous text chat in higher education (Shah-Nelson, 2013). In a platform such as WebEx, an instructor can use the integrated voice tool to talk about a particular topic while displaying a graphic, presentation, or document on the screen. Students can type questions or comments in the chat tool and can take turns talking when called upon. 
Audio, video, and text interactions all come with varying degrees of technical issues. When participating in an audio conference, speaker discomfort and uncertainty are not uncommon. The absence of audio feedback from a visible audience can make a speaker wonder: Is anyone else there? Can anyone hear me? Is the audience bored? As much as the technology can facilitate learning, it can also be a distracter (Branon \& Essex, 2001). For example, in a study of videoconferencing, Locatis et al. (2003) found that a technological delay affected speakers, resulting in delayed feedback from attendees. Even though the intent had been to mimic a seamless face-to-face environment, some of the participants' attention was diverted toward the technology. In a study about virtual classrooms, Falloon (2011) found that some students may have been distracted because they did not know how their audience perceived them or if they could be heard.

When teaching online via a synchronous medium, one must be prepared to account for qualitative differences in communication and feedback. The technology will have its own effect and presence on the learning context. Ideally, instructors will have anticipated any problems related to the technology and will be prepared with workarounds. When there is a speaker handoff (also known as a hand-over) or a transition from one speaker to another, the hope is that there will be no disruption in the overall conversation and that a smooth transaction will take place. In the event of a communication breakdown, however, all involved parties will naturally seek a way to resolve the problem.

In this study, I used a conversation analysis approach to analyze the moments when transitions from speaker to speaker occurred in an online synchronous course. Conversation analysis looks at everyday, naturally occurring interactions by analyzing not just the content of the conversation, but how the content is delivered by first transcribing the text and then marking up the text to include the structure and sequential patterns of the conversation, such as turn-taking between participants and repairs that occur in speech. The particular focus of this study is on whether or not the transitions were successful, due to technical problems (audio issues/Internet connectivity) or user errors (forgetting to unmute).

\section{Review of Related Literature}

\section{Turn-Taking in Natural Conversations}

To have a successful conversation, parties must be able to interact with each other and provide adequate information. They must be able to speak, hear, and understand.

The seminal research conducted by Sacks, Schegloff, and Jefferson (1974) analyzed the sequences between parties in natural conversations and described the interrelationships between the organizational structures. They found that one party talks at a time, the conversation goes back and forth between the parties (turn-taking), parties may be called upon to speak, there tends to be some overlap between parties, turns can be as short as one-word and not have a predetermined length, and nothing, such as the length of the conversation or number of turns, is fixed.

In their research, Sacks et al. (1974) identified three ways in which a change in speaker can be organized: the next speaker can be selected by the previous speaker, a speaker can self-select, or the present speaker can continue speaking. According to the authors, these three options are hierarchically organized: Other-selection goes before self-selection, which goes before continuation. 


\section{Turn-Taking in CMC Conversations}

Suggs, Dennen, and Myers (2013) found that the turn-taking rules identified by Sacks et al. (1974) still applied to the audio portion of the class, but the rules did not apply to the chat portion of the class. In Computer-Mediated Communication (CMC) that uses the chat feature, turn-taking is vastly different than it is in a face-to-face setting. The medium constrains how the flow of conversation goes (Garcia \& Jacobs, 1999; Herring, 1999; Markman, 2010; Schönfeldt \& Golato, 2003; Thorne, 2000). In a face-to-face conversation, there is typically one speaker at a time (Sacks, Schegloff, \& Jefferson, 1974) with a brief overlap. But in CMC, multiple parties can write and post messages at the same time in the chat window. Participants don't have any control over when a message will post to the chat window (Garcia \& Jacobs, 1999). The sequence in which the messages will display depends on how fast the parties are typing and how long the message is. Some parties may choose to type the entire message before pressing the Enter button versus typing a small chunk at a time in order to keep the conversation flowing without too long of a delay. If a party is taking too long to construct a message, the conversation may have already moved on to another topic (Markman, 2005). From a technical standpoint, there may be differences based on when the message was received by the server and the connection speed between the computer and the server (Rintel, Pittam, \& Mulholland, 2003).

Turns are constructed in isolation and the turn is only complete when the sender presses the Send or Enter button and the text is visible to the other parties (Markman, 2005). If there are several messages posted at one time, the respondent must determine which of the postings to first respond. Additionally, if a response is too far away from the original message it references, it may get lost in the stream of messages (González-Lloret, 2011). For these reasons, Garcia and Jacobs refer to this form of communication as "quasi-synchronous" (1999).

Using webconferencing software, like WebEx, enables participants to use the audio channel and the chat channel for turn-taking. A speaker can either call upon another party or another party can self-identify using the chat to indicate a desire to speak. The webconferencing software may also provide a way of letting the speaker know that someone else wants to speak. WebEx provides an icon with a hand (mimicking the classroom practice of hand-raising in order to signal a teacher). If a party clicks the icon, it indicates to the current speaker that the party wants to speak.

\section{Repairs in Conversations}

There are issues in conversations where the parties cannot hear or understand one another, or a party has difficulty speaking. When these issues or errors are identified and resolved, the process is known as a repair in conversation analysis (Schegloff, Jefferson, \& Sacks, 1977). Schegloff, Jefferson, and Sacks (1977) noted that sometimes a repair is found when there is no audible error or mistake. Conversely, audible errors or mistakes do not necessarily lead to repair. Moreover, the repair does not stand by itself. A cause resulting in repair is referred to as a "repairable" or "trouble source" (Schegloff et al., 1977), which is part of a larger repair sequence. First, a repair has to be initiated, perhaps by someone stating, "I can't hear you" (Egbert, 1997). After that has been stated, then a repair sequence takes place in order to resolve the issue. These repairs can seem somewhat seamless to us in the conversation because we have learned, at a very young age, that there are norms or rules for how to engage in a conversation and deal with a communication breakdown. 
The conversation analysis literature distinguishes between who initiates the repair and who executes the repair (Levinson, 1983). It is important to note than in a repair sequence, the person who initiates the repair "may not necessarily be the one who produces the repaired item" (Obeng, 1992, p. 63). In addition, when there are more than two people in a conversation, repair can be initiated by more than one speaker (Egbert, 1997).

There are four types of repair sequences. An example of repair in each case is provided below. The transcription markings typically found in conversation analysis studies are included.

1. Self-initiated self-repair: Repair is both initiated and carried out by the speaker of the trouble source.

$\mathrm{N}$ : She was givin' me a:1l the people that were go:ne this yea:r I mean this quarter y'//know (Schegloff et al., 1977, p. 364)

The speaker is speaking to another party and then quickly interrupts the other party to make the repair without prompting from the recipient. Lerner (1996) noted that the self-completed repair is the preferred method in conversations.

2. Other-initiated self-repair: Repair is carried out by speaker of the trouble source but is initiated by the recipient.

A: Have you ever tried a clinic?

B: What?

A: Have you ever tried a clinic? (Schegloff et al., 1977, p. 367)

The speaker is not clearly heard by the recipient, as is indicated by the question word. To repair the trouble source, the speaker repeats the same question as before.

3. Self-initiated other-repair: The speaker of a trouble source may try and get the recipient to repair the trouble. For instance, if a name is proving troublesome to remember.

B: hhh Well, I'm working through the Amfat Corporation.

A: The who?

B: Amfah Corporation. T's a holding company (Schegloff et al., 1977, p. 368)

The speaker may not know the name of the company and is expecting the recipient to provide the correct name. Instead, the recipient asks for clarification and the speaker makes the repair.

4. Other-initiated other-repair: The recipient of a trouble source both initiates and carries out the repair. This is closest to what is conventionally called a correction.

A: Lissena pigeons.

B: Quail, I think (Schegloff et al., 1977, p. 378)

The recipient makes the repair by correcting the error that the speaker made.

\section{Repairs in CMC}

Most frequently, repair sequences have been studied in the context of face-to-face or telephone conversations between two people. In face-to-face conversations, we assume that the person we are speaking with can hear us based on visual and auditory cues. In telephone conversations, we can no longer assume this is the case because there are no nonverbal cues to alert us that someone cannot hear us speaking. 
Studies of repair in telephone conversations (e.g. Egbert, 2004) more closely mimic audio conferencing interactions, although they also lack the additional text-based communication channel. In an online environment that does not use video, a speaker may have to ask the interlocutors "Can you hear me?" and then wait for a response from them either through the chat or through the audio channel. The silence during the pause does not necessarily mean that the interlocutors did not hear the speaker, but that the interlocutors are typing, waiting, editing, or reading before responding (Garcia \& Jacobs, 1999).

Conversation analysis research in a synchronous online environment has primarily looked at conversational repair in chat rooms (González-Lloret, 2011; Markman, 2010; Schönfeldt \& Golato, 2003). Schönfelt and Golato (2003) examined a German Web chat and found that participants applied the same behaviors used in oral conversations to the chat. If there was a trouble source, the conversation is stopped to address the trouble source. The same preference for self-repair that is found in oral conversations was found for the chat medium. Markman's (2006) research also noted the preference for self-repair in chat, most typically for a typo or spelling correction. Additionally, Schönfeldt and Golato (2003) found that the most frequent type of repair was the other-initiated self-repair, followed by self-initiated self-repair.

\section{Purpose of the Study}

The purpose of the study is to identify when repair sequences take place and who initiates the repair sequence and through what channel (chat or voice or both). Although there has been extensive research on conversation analysis and repair sequences in traditional single-channel settings (e.g. telephone conversations or face-to-face conversations), this study will apply conversation analysis to a dual-channel setting (audio and chat) to identify technical difficulties in an online class and how those two channels interact together so the conversation can be repaired.

In a review of the literature on conversation analysis studies in online talk, Paulus, Warren, and Lester (2016) found that the majority of the studies were published in language communication journals. Only three out of 89 studies appeared in education journals and 10 out of 89 studies focused on repair. Most of the studies focused on sequence organization, turn-design, or turn-taking. This study intends to use conversation analysis in an online synchronous educational setting.

The research questions guiding the study are:

1. Are speaker hand-offs smooth?

2. When are repair sequences taking place?

3. Who initiates the repair sequence?

4. Who is handling the repairs and through what channel?

\section{Participants}

\section{Methods}

Participants in this study were the instructor and 16 graduate students (including myself) in a discourse and conversation analysis class at a large, public university in the southeastern United States. There were 14 females (including the instructor) and 3 males. All participants consented to be included in the study. The instructor for the class had some experience teaching 
in an online synchronous environment and about one-half of the students had prior experience in an online course, mostly in an asynchronous environment.

\section{Course Format}

The graduate-level course in discourse and conversation analysis was taught using a blended format, with both face-to-face meetings and online synchronous sessions using WebEx. WebEx is a videoconferencing software application that enables participates to listen and talk to each other, view the moderator's (either the instructor or a student) screen when the moderator is sharing a presentation, and type in a chat tool. To hear the audio, participants can either use their speakers and microphone through their computer or call a given WebEx number and use a passcode.

The course was based around discussions about the readings and mini-experiments that the students conducted to reinforce the readings. This study focuses only on the seven WebEx synchronous class sessions from weeks 2, 3, 5, 7, 8, 10, and 11. Each WebEx session lasted about 90 minutes.

The instructor would begin the class by telling the students to mute their audio (to prevent audio feedback) and then would present an agenda on the WebEx screen. As the instructor discussed a particular topic for the day, students would ask questions or make comments in the chat session. If students wanted to speak, they would identify themselves by "raising" their hand (by clicking a hand icon on the WebEx screen) or typing something in the chat window and then the instructor would call on the individual. At that point, the student would unmute and begin speaking.

\section{Data Collection Methods}

Each class session (both face-to-face and online) was recorded. All participants agreed that the sessions could be recorded. Data were collected from the recorded audio, as well as the saved chat transcripts, for each of the seven online WebEx sessions. WebEx saves the public chat transcripts and the audio for playback.

\section{Data Analysis}

I used a conversation analysis approach (Goodwin, 1981; ten Have, 1999; Schegloff, 2007; Schegloff et al., 1977; Wooffitt, 2005) to analyze the data. Conversation analysis looks at the structure and sequential patterns of conversation, such as turn-taking between participants, overlapping speech between participants, utterances in speech, pauses during speech, and repairs that occur in speech. In traditional transcriptions, these patterns are not noted. However, they are the foundation for conversation analysis research.

In order to analyze the data, I first read through the chat transcripts of the seven online WebEx sessions to quickly identify when there was an attempt at a speaker hand-off. I looked for words of affirmation from the participants. This could be identified by several "yes" or "I can" responses. I also looked for areas where participants stated an issue with hearing the speaker, such as "I can't hear you" or several "no" responses by various participants. To confirm these exchanges and to locate any additional speaker hand-offs, I then listened to the corresponding audio files to verify that there was a trouble source. Where there was an attempt at a speaker handoff, I transcribed those sections of the audio. Finally, I matched the audio transcript to the chat transcript to see how the trouble source was repaired (and through which channel) and then 
indicated if there were pauses in the audio. I used pseudonyms in reporting the data in order to preserve the identities of the students and the instructor.

\section{Results}

Throughout the WebEx sessions, speaker hand-offs became a common occurrence. Sometimes those transitions were smooth and other times they were not. There were several instances in which a problem or trouble source took place. In these cases, how the repair was made and by whom varied. In addition, other technical issues occurred throughout the WebEx sessions, such as when parties were having audio or Internet connectivity issues.

\section{Turn-Taking}

In this study, there were several different approaches to taking the lead in the conversation. In many of the cases, the called-upon student would enter the conversation asking if he could be heard. The student would then receive confirmation either through the audio channel or the chat or both channels before proceeding. In one instance, there was a slight delay in receiving the chat confirmation. In this case, the speaker had begun speaking after receiving the audio confirmation and did not wait for confirmation in the chat window. In these cases, there were no trouble sources and no need for a repair sequence.

Chat. The called-upon speaker asks if she could be heard. She then receives confirmation through the chat before proceeding (Table 1).

\begin{tabular}{lll}
\hline Speaker & Audio Channel & Chat Channel \\
\hline Instructor & I'm passing the ball over to Shay. & - \\
& Okay, you guys can unmute & \\
& yourselves when you are ready \\
& to talk. \\
& Okay, good morning everybody. & - \\
Shay & So, everybody can hear me? & \\
& - & yes \\
Instructor & - & yes \\
Naomi & - & yes \\
Ally & - & yes \\
Brandi & - & ya \\
Alexis & - & Good morning! \\
Katherine & - & yes \\
Angela & - & Yes \\
Andres & - &
\end{tabular}

Table 1. Hand-Off with Confirmation Through Chat Channel, from Week 7 
In this case, there were no issues with speaker hand-off. The instructor states that she is calling on Shay and Shay confirms she is heard before moving on with her response. Several students and the instructor confirm through the chat channel that they can hear her.

Audio. The called-upon student asks if he could be heard. He then receives audio confirmation before proceeding (Table 2).

\begin{tabular}{lll}
\hline Speaker & Audio Channel & Chat Channel \\
\hline Deanna & Go ahead Andres. Introduce & - \\
& yourself. \\
Andres & Ah. Can the group hear me? & - \\
Deanna & I can hear you. & - \\
Andres & (continues speaking) & - Channel, from Week 8
\end{tabular}

In this case, Deanna calls upon Andres. Andres asks for confirmation that he can be heard and receives confirmation from Deanna (the prior speaker) through the audio channel before he continues.

Dual channel. The called-upon student asks if she can be heard. She then receives confirmation through both the chat and the audio channels before proceeding (Table 3 ).

\begin{tabular}{lll}
\hline Speaker & Audio Channel & Chat Channel \\
\hline Instructor & Okay, Naomi, I see you have your & - \\
& hand up. Why don't you unmute & \\
& and chime in too. \\
Naomi & Does this work? Can you hear me? & - \\
Instructor & I can hear you. & - \\
Deanna & - & yes working!
\end{tabular}

Table 3. Hand-Off with Confirmation Through Both Channels, from Week 7

The instructor calls upon Naomi to speak. Naomi asks if she can be heard through the audio channel and receives confirmation from the instructor in the audio channel and from another student in the chat channel.

None requested. The speaker assumes that everyone could hear and begins leading the discussion prior to receiving any confirmation through the chat (Table 4). 
Turn-taking in both channels. A speaker identifies herself as being the next speaker in the chat channel and then asks for confirmation in the audio channel (Table 6).

\begin{tabular}{lll}
\hline Speaker & Audio Channel & Chat Channel \\
\hline Deanna & You want to take that Naomi, or & - \\
& anybody? & i can \\
Naomi & - & - \\
Naomi & Can everyone hear me? & - \\
Deanna & You sound great. & - \\
Naomi & Oh good, it finally works. & yes \\
Katherine & - & yes \\
Kim & - & yes Naomi \\
Ally & - & -
\end{tabular}

Table 6. Turn-Taking Using Both Channels, from Week 8

In this case, speaker hand-off occurs using both channels, for two distinct purposes. First, Deanna calls upon Naomi. Then, Naomi identifies herself as the next speaker in the chat channel and uses the audio channel to confirm that she can be heard. She receives confirmation in both channels.

Note in each of these cases that the practice of asking "Can you hear me?" is one in which the only possible confirmation is an affirmative one. Confirmation was requested most often when a speaker entered the audio conference for the first time during that session or after a prolonged silence. Thus, it became a regular part of the initiation sequence for most speakers, and the class became used to conversations that were interrupted with checks of audio functionality during major turn-taking moments.

\section{Repairs}

There were situations in which a speaker who was not heard would not yield a reply, or at least not until someone experienced an awkward silence. Such silences could result in either selfor other-initiated repairs. There were two main repair scenarios that occurred during the study: fixing the problem and moving on. A hybrid form of attempting or allowing a fix and then moving on also occurred. Below are five examples of when they were used:

Fixing the problem. A speaker identifies that there is an issue and eventually fixes the problem through the audio channel (Table 7).

\begin{tabular}{lll}
\hline Speaker & Audio Channel & Chat Channel \\
\hline Instructor & So, Naomi, you said this happened - \\
& $\begin{array}{l}\text { to you. Do you want to share the } \\
\text { experience? You don't have to, I } \\
\text { just thought you might want to } \\
\text { talk about it. }\end{array}$ \\
\hline
\end{tabular}




\begin{tabular}{|c|c|c|}
\hline Naomi & - & hope my mic works \\
\hline Instructor & $\begin{array}{l}\text { Just unmute yourself and give it } \\
\text { a shot. }(8 \mathrm{sec}) \text { If you're talking } \\
\text { we're not hearing you. }(12 \mathrm{sec})\end{array}$ & - \\
\hline Angela & - & I can hear her \\
\hline Instructor & Nope we still, you can hear her? & - \\
\hline Naomi & - & just a sec \\
\hline Angela & - & coz she is sitting right next to me \\
\hline Wendy & - & I can't hear her \\
\hline Kim & - & i can't \\
\hline Andres & - & mine is not working aswell. \\
\hline Wendy & - & haha \\
\hline Angela & $\begin{array}{l}\text { Okay, so Naomi's going to use } \\
\text { my microphone cuz her's is not } \\
\text { working. }\end{array}$ & - \\
\hline Instructor & That's very kind of you Angela & - \\
\hline Naomi & Okay, so people can hear me? & - \\
\hline Instructor & Yes. & - \\
\hline Naomi & Okay, good [continues talking] & - \\
\hline Ally & - & yes \\
\hline Tamara & - & yes \\
\hline Cheyenne & - & yes... \\
\hline Wendy & - & Yep \\
\hline
\end{tabular}

Table 7. Fixing the Problem Using Audio Channel, from Week 7

In this example of an other-initiated self-repair, the instructor called upon a student (Naomi) and then waited for 8 seconds, then another 12 seconds for Naomi to respond. Angela responded that she could hear Naomi (she was in the same room) and was going to let her use her microphone. The entire exchange took nearly a minute before Naomi was able to respond through Angela's microphone.

A speaker can also identify that there is a problem and the problem is resolved through the chat channel (Table 8). 


\begin{tabular}{lll}
\hline Speaker & Audio Channel & Chat Channel \\
\hline Alex & - & can you hear me? \\
Ally & - & no \\
Naomi & - & no \\
Brian & - & me either \\
Cheyenne & - & can't hear you \\
Lindsay & - & no \\
Alex & - & ok \\
Alex & - & let me type
\end{tabular}

Table 8. Fixing the Problem Using the Chat Channel, from Week 10

In this example of a self-initiated, self-repair, there is no indication that Alex cannot be heard until he asks through the chat channel. We can assume that Alex must have identified that he was not being heard by asking, "Can you hear me" through the audio channel. When he did not receive a response through either channel, he typed the same question in the chat channel. In order to correct the issue, he proceeds by using the chat channel to type his response.

Allowing a fix, then moving on. The speaker allows for a fix but can also move on if there is an indication that the problem cannot be resolved (Table 9).

\begin{tabular}{lll}
\hline Speaker & Audio Channel & Chat Channel \\
\hline Instructor & $\begin{array}{l}\text { Let's start with MaryAnne. What } \\
\text { do you think MaryAnne? Don't } \\
\text { forget to take your phone off mute. }\end{array}$ & - \\
& $\begin{array}{l}\text { (20 sec.) Can anyone hear } \\
\text { MaryAnne? I can't you MaryAnne }\end{array}$ & \\
& unfortunately. & I am speaking but no one can hear \\
MaryAnne & - & no \\
Angela & - & no \\
Tamara & - & no \\
Ally & - & no \\
Deanna & - & no \\
Cheyenne & - & no \\
Wendy & - & nope \\
Naomi & - & no \\
Lindsay & - &
\end{tabular}




\begin{tabular}{ll}
\hline Instructor & Okay, we're going to have to \\
& figure out your audio at some \\
& point MaryAnne, but um in the \\
meantime you can type in the \\
chat. I'm going to call on. I think \\
Deanna had a hand up. Deanna, \\
do you want to reply to this?
\end{tabular}

Table 9. Allowing a Fix, Then Moving On, from Week 2

In this case, the trouble source came from the instructor who indicated she couldn't hear MaryAnne. MaryAnne attempts to initiate the repair by typing that she is speaking. Eventually, the instructor has to complete the repair by calling on another student. The instructor has already waited 20 seconds for MaryAnne to begin speaking and has chosen to not spend more time waiting for MaryAnne to be heard. This case is a self-initiated self-repair because the instructor identifies the trouble source, attempts to correct it, and resolves it. The repair is resolved when the instructor chooses to move on to another student.

Moving on. Another response is not waiting for a confirmation from the other party after a trouble source has been indicated. The result is simply to move on with the conversation (Table $10)$.

\begin{tabular}{lll}
\hline Speaker & Audio Channel & Chat Channel \\
\hline Deanna & Brian, I remember Gladiator, but I \\
& don't remember his introduction. \\
& Do you want to tell us about that \\
& (3 sec) or write it? Does anybody \\
& remember how he identified \\
& himself? (9 sec) Well, I'm sorry I \\
& can't hear you but I'm going to move \\
& on. Please don't take offense. \\
& [continues talking] \\
Brian & - & my mic is not working
\end{tabular}

Table 10. Moving On Without Confirmation from Other Party, from Week 8

Deanna attempts the hand-off to Brian, but moves on with the discussion after not receiving a verbal or typed response from him. In this case, the speaker only waited a few seconds after attempting the speaker hand-off before continuing on with the discussion. She moves on without allowing the other person to identify that there is a problem. This is another case of a self-initiated self-repair because the speaker identifies the trouble source, attempts to correct it, and resolves it. The repair is resolved when the speaker chooses to move on with the discussion.

Troubleshooting, then moving on. A student called upon another student but isn't able to hear her. The instructor and the speaker try to troubleshoot the situation before the speaker moves on without a confirmation from the called-upon speaker (Table 11). 


\begin{tabular}{lll}
\hline Speaker & Audio Channel & Chat Channel \\
\hline Deanna & Anybody? Okay, Katherine. & - \\
& $\begin{array}{l}\text { You want to unmute yourself } \\
\text { Katherine and contribute. }\end{array}$ & \\
Instructor & I'm wondering if Katherine's & - \\
& microphone isn't working again. & \\
Deanna & Yeah, I'm wondering. & - \\
Instructor & We can't hear you Katherine & - \\
Deanna & [moves on with the discussion] & - \\
Katherine & - & guess my mic is broken again. \\
\hline
\end{tabular}

Table 11. Troubleshooting, Then Moving On, from Week 8

Again, the speaker doesn't wait for the other person to identify there is a problem before moving on. It's also interesting to note that the speaker has acquired the behavior of the instructor by telling the speaker to unmute herself before contributing and that the speaker and the instructor are both trying to fix the problem.

\section{Technical Issues}

There are also instances in which participants need technical help due to their audio not working or they are experiencing Internet connectivity issues.

Audio issues. In the following example, the instructor is still leading the discussion through the audio channel, but the chat channel is active with participants trying to troubleshoot the audio problems. At one point, the instructor also makes use of the chat channel to troubleshoot the audio (Table 12).

\begin{tabular}{|c|c|c|}
\hline Speaker & Audio Channel & Chat Channel \\
\hline Instructor & [Leading a discussion] & - \\
\hline Kim & - & Is anyone speaking? \\
\hline Naomi & - & Instructor is speaking \\
\hline Deanna & - & clear here \\
\hline Ally & - & $\begin{array}{l}\text { For those who can't hear: There is a mute } \\
\text { button for both the speaker volume and } \\
\text { microphone. Make sure the mute box for } \\
\text { speaker is not checked. }\end{array}$ \\
\hline Lindsay & - & I cannot hear anything yet... \\
\hline Brian & - & me neither \\
\hline Instructor & [continues instructing] & you need to join the audio \\
\hline
\end{tabular}




\begin{tabular}{lll}
\hline Naomi & - & $\begin{array}{l}\text { Lindsay and Brian need to either call the } \\
\text { phone \# or join with computer headset }\end{array}$ \\
Kim & - & I can hear clearly now! \\
Brian & - & i can hear you
\end{tabular}

Table 12. Troubleshooting Audio Issues in the Chat Channel, from Week 2

The previous example is from the first WebEx session during week 2 of the semester. It is possible that participants are still working on the technical aspects of using WebEx. However, issues similar to this occurred throughout the semester in week 5 and week 8 , as can be seen in the following examples.

As shown in Table 13, Wendy wants to participate in the audio channel but is unable to do so because her microphone is not working. This example is from week 5 of the semester.

\begin{tabular}{lll}
\hline Speaker & Audio Channel & Chat Channel \\
\hline Wendy & - & $\begin{array}{l}\text { My mic has not worked the last two times, } \\
\text { so raising my hand is like false } \\
\text { advertising... :-( }\end{array}$ \\
Kim & - & Oh no Wendy :)
\end{tabular}

Table 13. Technical Issue with Microphone, from Week 5

Similarly, Brian indicates he also wants to participate in the audio channel, but his microphone is also not working (Table 14).

\begin{tabular}{lll}
\hline Speaker & Audio Channel & Chat Channel \\
\hline Brian & - & if my mic worked, I would raise hand \\
& & several times already \\
\hline
\end{tabular}

Table 14. Technical Issue with Microphone, from Week 8

Although Wendy and Brian were having technical difficulties with their microphones, they could have still participated in the discussion through the chat channel as Alex did (Table 8). They may have chosen not to participate through the chat channel because of the length of time it would take to type out their comments.

In another instance, Deanna is losing her audio through the built-in feature in WebEx. Ally recommends that she call in to WebEx (Table 15).

\begin{tabular}{lll}
\hline Speaker & Audio Channel & Chat Channel \\
\hline Deanna & - & keep losing audio \\
Ally & - & no \\
\hline
\end{tabular}




\begin{tabular}{lll}
\hline Deanna & - & ok now \\
Tamara & - & no \\
MaryAnne & - & no \\
Cheyenne & - & nope \\
Ally & - & call in Deanna \\
\hline
\end{tabular}

Table 15. Technical Issue with Losing Audio, from Week 10

Internet. In a synchronous environment, Internet connectivity is critical for being able to participate in the class. In the following example (Table 16), Brian is leading a discussion and then there is silence. The chat channel is used to let him know that he can no longer be heard.

\begin{tabular}{lll}
\hline Speaker & Audio Channel & Chat Channel \\
\hline Brian & [Leading a discussion, then silence $]$ & - \\
Cheyenne & - & can't hear you... \\
Deanna & - & lost audio \\
Angela & - & cant hear either \\
Brian & HELLO? & - \\
Angela & - & now I an ehre \\
Deanna & - & ok \\
Cheyenne & - & yes... now I can.. \\
Lindsay & - & we can hear u \\
MaryAnne & - & can hear now \\
Andres & - & ok \\
Brian & Hello, can you hear me? & - \\
Instructor & - & yes \\
Kim & - & yes \\
Lindsay & - & yes! \\
MaryAnne & - & yes \\
Brian & Good. I just uh lost connection & - \\
& of the Internet so I thought that & \\
I'd...I resume. I resume. & \\
Deanna & - & yes, can hear you now \\
\hline Table 16. Technical Issue with Internet Connectivity, from Week 11 &
\end{tabular}




\section{Discussion}

The purpose of the study was to identify when repair sequences took place and who initiated the repair sequence and through what channel (chat or voice or both). All of these examples illuminate the types of technical and conversational problems that may hinder communication - even if just temporarily-in a synchronous learning session. Each time there was a speaker hand-off, the speaker seemed to anticipate if an issue would occur. The speaker would tell the next speaker to unmute before speaking. This occurrence is unique to the online environment because the medium constrains how the flow of conversation goes (Garcia \& Jacobs, 1999; Herring, 1999; Markman, 2010; Schönfeldt \& Golato, 2003; Thorne, 2000).

In most instances, speaker hand-offs went smoothly. The instructor informed students that they could use the chat or raise a hand, using the hand icon in WebEx, to speak next. Then the instructor would call upon a specific party or another party would self-identify as the next speaker either through raising a hand or typing in the chat channel. The entering party would ask if the audio was working. After receiving confirmation from others through the audio channel, the chat channel, or both channels, the entering party would continue speaking. The students modeled this same behavior as they were calling upon another party to speak. This confirms research by Hutchby (2001), who refers to specific "norms of behavior," or essentially how to appropriately participate in the community.

However, there were instances in which the speaker hand-offs encountered a trouble source. The speaker would call upon a specific party and then wait during the silence. As Garcia and Jacobs (1999) noted, the silence during the pause does not necessarily mean that the interlocutors did not hear the speaker, but that the interlocutors are typing, waiting, editing, or reading before responding. There were many instances in which this silence occurred. During this period, the speaker would have to determine whether or not there was an issue with the calledupon speaker's audio. If there was a trouble source, the conversation stopped to address it. These findings are similar to Schönfelt and Golato (2003). The current speaker could either try to troubleshoot the issue, repeat the request, continue to wait, or move on.

When there were hand-off issues, using the chat channel became a useful way of troubleshooting or indicating that there was an issue. If someone lost audio, the person could still communicate through the chat channel in order to resolve or repair the issue. These findings were similar to Martin, Parker, and Deale (2012) in which the chat channel provided a way for parties to communicate technical difficulties.

The role of the instructor is also important in an online class. Instructors may serve as facilitators, coaches or guides (Bonk, Wisher, \& Lee, 2003; Lee, Lee, Liu, Bonk, \& Magjuka, 2009; Liu, Bonk, Magjuka, Lee, \& Su, 2005). Moreover, they may also serve in a managerial or a technical role (Berge, 1995; Bonk, Kirkley, Hara, \& Dennen, 2001). In a face-to-face classroom, instructors have the right to talk at any given time and to any other person. If there is some gap in the conversation, instructors can also fill the silence or interrupt a speaker as needed (Cazden, 2001). This was seen in many of the examples. The instructor's role was to troubleshoot, call upon students, and move the discussion along. Current speakers could also choose to play this role. In Tables 2, 6, 10 and 11, the speaker took on the role of the instructor by calling upon other speakers. Additionally, the instructor seemed to be more tolerant of waiting and troubleshooting, as opposed to other speakers who moved on without waiting for a response (Tables 10 and 11). 
Throughout the semester, the instructor also managed both channels and integrated them into the discussion. Using the text channel and the audio channel are helpful for students to receive immediate feedback (Martin \& Parker, 2014). Vu and Fadde (2013) found that students were happy to use the chat channel as a pedagogical method, but the information needed to be addressed right away by the instructor. Pullen (2004) noted that students used the audio channel when they were telling a long story and used the chat channel when they had a brief comment. Therefore, it is important that instructors keep up with the chat channel to validate the significance of communication occurring there (Martin, Parker, \& Deale, 2012).

\section{Limitations}

A limitation of this research is the inability for researchers to generalize the findings. This course was also a discussion-based graduate level course. Many of the students had taken an online synchronous course before, were familiar with the instructor, and were willing to speak-up or type in the chat when someone else was talking. This may not be the case with a different group of students.

In addition, this study did not analyze data based on what happened after the successful or unsuccessful hand-off. This study focused only on analyzing the structure of the conversation, as opposed to the discourse. However, future studies could address whether or not the hand-off (either successful or unsuccessful) leads to a more engaging discussion.

\section{Implications and Future Research}

This study provides some insight on how chat can be used in a discussion-based, online synchronous course to identify technical difficulties when a speaker is called upon and how corrections are made. The instructor and students need to be prepared for someone who is having technical difficulties hearing or speaking. The chat channel can provide an additional layer of support to work through technical issues.

Instructors who teach online synchronous courses need to be comfortable teaching in an environment that uses two different channels (chat and audio) and learn how to manage the two channels together. Instructors also need to be able to read through the chat comments and provide feedback based upon the comments. The chat channel provides an additional level of interaction in the course.

Ideally, the instructor should communicate ground rules at the beginning of the course. Ground rules could include things like hand-raising to identify students who want to speak and when students should place their audio on mute. Perhaps everyone could conduct an audio check at the beginning of the class to ensure that everyone can hear and speak. Instructors could also identify what the purposes are for the audio and chat channels. Having ground rules could preemptively avoid technical difficulties and ensure the course runs smoothly.

Future researchers need to examine additional classes using a variety of factors, including those with different class sizes, different instructors outside of this field, and different levels of instructor familiarity with online environments. More studies using conversation analysis in an online synchronous environment should be conducted to analyze naturally occurring interactions between teacher-student and student-student. 
Navigating Turn-Taking and Conversational Repair in an Online Synchronous Course

\section{Author Note}

Data for this study were collected while the author was a graduate student at Florida State University.

Correspondence concerning this article should be addressed to Yvonne Earnshaw. E-mail: yvonne.earnshaw@gmail.com 


\section{References}

Berge, Z. (1995). Facilitating computer conferencing: Recommendations from the field. Educational Technology, 35(1), 22-30.

Bonk, C. J., Kirkley, J. R., Hara, N., \& Dennen, V. (2001). Finding the instructor in postsecondary online learning: Pedagogical, social, managerial, and technological locations. In J. Stephenson (Ed.), Teaching and learning online: Pedagogies for new technologies (pp. 76-97). London: Kogan Page.

Bonk, C. J., Wisher, R. A., \& Lee, J-Y. (2003). Moderating learning-centered e-learning: Problems and solutions, benefits and implications. In T. S. Roberts (Ed.), Online collaborative learning: Theory and practice (pp. 54-85). Hershey, PA: Idea Group Publishing. doi:10.4018/978-1-59140-174-2.ch003

Branon, R. F., \& Essex, C. (2001). Synchronous and asynchronous communication tools in distance education. Tech Trends, 45(1), 36-42.

Cazden, C. B. (2001). Classroom discourse: The language of teaching and learning. Portsmouth, NH: Heinemann.

Egbert, M. (1997). Some interactional achievements of other-initiated repair in multiperson conversation. Journal of Pragmatics, 27, 611-634.

Egbert, M. (2004). Other-initiated repair and membership categorization: Some conversational events that trigger linguistic and regional membership categorization. Journal of Pragmatics, 36(8), 1467-1498.

Falloon, G (2011). Exploring the virtual classroom: What students need to know (and teachers should consider). Journal of Online Learning and Teaching, 7(4), 439-451.

Garcia, A. C., \& Jacobs, J. B. (1999). The eyes of the beholder: Understanding the turn-taking system in quasi-synchronous computer-mediated communication. Research on Language and Social Interaction, 32(4), 337-367. doi:10.1207/S15327973rls3204_2

González-Lloret, M. (2011). Conversation analysis of computer-mediated communication. CALICO Journal, 28(2), 308-325.

Goodwin, C. (1981). Conversational organization: Interaction between speakers and hearers. New York, NY: Academic Press.

Have, P. ten (1999). Doing conversation analysis: A practical guide. London, England: Sage.

Herring, S. C. (1999). Interactional coherence in CMC. Journal of Computer-Mediated Communication, 4. doi: 10.1111/j.1083-6101.1999.tb00106.x

Hutchby, I. (2001). Conversation and technology: From the phone to the Internet. Cambridge, UK: Polity.

Lee, S.-H., Lee, J., Liu, X., Bonk, C. J., \& Magjuka, R. J. (2009). A review of case-based learning practices in an online MBA program: A program-level case study. Educational Technology \& Society, 12(3), 178-190. 
Lerner, G. H. (1996). Finding "face" in the preference structures of talk-interaction. Social Psychology Quarterly, 59(4), 303-321.

Levinson, S. C. (1983). Pragmatics. Cambridge, UK: Cambridge University Press.

Liu, X., Bonk, C. J., Magjuka, R. J., Lee, S-h., \& Su, B. (2005). Exploring four dimensions of online instructor roles: A program level case study. Journal of Asynchronous Learning Networks, 9(4), 29-48.

Locatis, C., Fontelo, P., Sneiderman, C., Ackerman, M., Uijtdehaage, S., Candler, C., . . . Dennis, S. (2003). Webcasting videoconferences over IP: A synchronous communication experiment. Journal of the American Medical Informatics Association, 10, 150-153. doi:10.1197/jamia.M1170

Markman, K. M. (2005). To send or not to send: Turn construction in computer-mediated chat. In C. Sunakawa, T. Ikeda, S. Finch \& M. Shetty (Eds.), Proceedings of the Twelfth Annual Symposium About Language and Society-Austin, 48 (pp. 115-124). Austin, TX: Texas Linguistic Forum.

Markman, K. M. (2006). Computer-mediated conversation: The organization of talk in chatbased virtual team meetings (Doctoral dissertation). Available from ProQuest Dissertations and Theses database. (UMI No. 3244348)

Markman, K. M. (2010). Learning to work virtually: Conversational repair as a resource for norm development in computer-mediated team meetings. In J. Park and E. Abels (Eds.), In Interpersonal relations and social patterns in communication technologies: Discourse norms, language structures and cultural variables (pp. 220-236). Hershey, PA: IGI Global. doi:10.4018/978-1-61520-827-2.ch012

Martin, F., \& Parker, M. A. (2014). Use of synchronous virtual classrooms: Why, who, and how? Journal of Online Learning and Teaching, 10(2), 192-210.

Martin, F., Parker, M. A., \& Deale, D. F. (2012). Examining interactivity in synchronous virtual classrooms. The International Review of Research in Open and Distributed Learning, $13(3), 228-261$.

Obeng, S. G. (1992). A phonetic description of some repair sequences in Akan conversation. Text, 12(1). 59-80.

Paulus, T., Warren, A., \& Lester, J. N. (2016). Applying conversation analysis methods to online talk: A literature review. Discourse, Context \& Media, 12, 1-10. doi:10.1016/j.dcm.2016.04.001

Pullen, J. M. (2004). Synchronous internet distance education: Wave of the future or wishful thinking? Proceedings from the E-technologies in Engineering Education Conference 2002. Davos, Switzerland.

Rintel, E. S., Pittam, J., \& Mulholland, J. (2003). Time will tell: Ambiguous non-responses on Internet Relay Chat. Electronic Journal of Communication, 13(1). Retrieved from http://www.cios.org/EJCPUBLIC/013/1/01312.HTML 
Sacks, H., Schegloff, E. A., \& Jefferson, G. (1974). A simplest systematics for the organization of turn-taking for conversation. Language, 50(4), 696-735. doi:10.2307/412243

Schegloff, E. A. (2007). Sequence organization in interaction: A primer in conversation analysis. Cambridge, UK: Cambridge University Press.

Schegloff, E. A., Jefferson, G., \& Sacks, H. (1977). The preference for self-correction in the organization of repair in conversation. Language, 53(2), 361-382. doi:10.2307/413107

Schönfeldt, J., \& Golato, A. (2003). Repair in chats: A conversation analysis approach. Research on Language and Social Interaction, 36(3), 241-284.

Shah-Nelson, C. (2013). Synchronous tools in support of teaching and learning. In Y. Kats (Ed.), Learning management systems and instructional design: Best practices in online education (pp. 172-191). Hershey, PA: IGI Global.

Suggs, C. L., Dennen, V. P., \& Myers, J. B. (2013). Juggling channels and turn-taking in a dual channel synchronous course: A conversation analysis approach. In S. Wang \& H. Yang (Eds.), Cases on Formal, Non-Formal, and Informal Online Learning: Opportunities and Practices (pp. 305-322). Hershey, PA: IGI Global. doi:10.4018/978-1-4666-19364.ch016

Thorne, S. (2000). Beyond bounded activity systems: Heterogeneous cultures in instructional uses of persistent conversation. In S. Herring \& T. Erickson (Eds.), Proceedings of the Thirty-Third Annual Hawaii International Conference on System Sciences. Los Alamitos: IEEE Computer Society.

Vu, P., \& Fadde, P. J. (2013). When to talk, when to chat: Student interactions in live virtual classrooms. Journal of Interactive Online Learning, 12(2), 41-52.

Wooffitt, R. (2005). Conversation analysis and discourse analysis: A comparative and critical introduction. London, England: Sage. 